\title{
Office Hysteroscopy in Difficult Embryo Transfer; Case Report of Three Cases
}

\author{
Mohamed Elmahdy', Hoda F. Abdel Salam², Nehal A. Kobeisy ${ }^{3}$ \\ ${ }^{1}$ Department of Obstetrics and Gynecology, Faculty of Medicine, Alexandria University, Alexandria, Egypt \\ ${ }^{2}$ Department of Obstetrics and Gynecology, Ministry of Health Hospitals, Alexandria, Egypt \\ ${ }^{3}$ Madina Fertility Centre, Alexandria, Egypt \\ Email: mahdy_moh@yahoo.com
}

Received 25 November 2015; accepted 28 February 2016; published 2 March 2016

Copyright (C) 2016 by authors and Scientific Research Publishing Inc.

This work is licensed under the Creative Commons Attribution International License (CC BY).

http://creativecommons.org/licenses/by/4.0/

(c) (i) Open Access

\begin{abstract}
Objectives: To facilitate embryo transfer technique in cases with previous failed or difficult transfer using office hysteroscopy. Study Design: Case report of 3 cases of previous difficult embryo transfer (ET). Methods: Hysteroscopic assisted insertion of cervical stent at the end of withdrawal bleeding in the stimulation cycle. This stent was used as a track for embryo transfer catheter. Results: One case cancelled before (embryo transfer) ET and the other 2 cases didn't get pregnant in spite of easy embryo transfer. Conclusion: This hysteroscopic assisted ET technique may facilitate ET technique in cases with difficult embryo transfer due to deformed cervix.
\end{abstract}

\section{Keywords}

\section{Office Hysteroscopy, Embryo Transfer, ICSI}

\section{Introduction}

Embryo transfer is a crucial step in the process of (intra cytoplasmic sperm injection) ICSI. Systematic reviews have shown that US guidance improves the clinical pregnancy, ongoing pregnancy, and live-birth rates in women when compared with the standard clinical touch method [1]-[4]. Ultrasound helps in confirming the position of the catheter tip within the uterine cavity, properly determining the site of embryo deposition, and decreasing the frequency of “difficult” ETs [2]. Difficult embryo transfers significantly diminish the pregnancy and implantation rates in patients undergoing ICSI [5]. Also, difficult embryo transfer was found to be associated with higher incidence of ectopic pregnancy than cases with easy transfer especially when the patient has a history of tubal damage or previous EP [6]. The problem behind difficult ET in most studies was found to be due to acute flexion or deflexion of the cervical canal or internal trauma to the endocervix or the endometrium resulted from 
previous surgeries, infection or mock ET. Cervical stenosis wasn't a finding in these cases [7].

Different maneuvers were tried to overcome difficult transfer; when patent tubes are present, zygote intrafallopian transfer is an excellent way in cases of failed or difficult cervical transfer [8]. Laminaria tent was used to help in cervical dilatation before ET [9] and cervical dilatation using Hegar dilators also was used in cases with cervical stenosis [10]. Hysteroscopy played a role in these cases such as hysteroscopic examination and placement of a Malecot catheter is a useful technique that allows easier transcervical ET in cases that have been difficult. This procedure may lead to improved pregnancy rates [11]. Hysteroscopic cervical canal shaving and preparation before transfer also, were done also to facilitate transfer [12]. Hysteroscopic guided embryo transfer was successful in some cases [13].

\section{Objectives}

Facilitate embryo transfer technique in cases with previous failed or difficult transfer using office hysteroscopy.

\subsection{Study Design}

Case report of 3 cases of previous difficult embryo transfer due to abnormal cervical canal associated with irregular track or acute angulation of the cervix.

\subsection{Participants/Methods}

2 cases of previous difficult embryo transfer and one case of cancelled transfer and all embryos were cryo preserved.

The first case was 37-year-old complaining of secondary infertility 10 years after first trimester abortion. She had 3 trials of ICSI/ ET but were unsuccessful. The last two trials were very difficult and under general anesthesia with prolonged maneuver. Office hysteroscopy was done before the last trial and revealed abnormal cervical canal track. In this trial she had very easy ET for one embryo (4A) on day 3.

The second case is 39-year-old with previous failed one trial of ICSI due to failed embryo transfer and three embryos were cryo preserved. Office hysteroscopy was done and revealed fibrosed cervical canal with irregular track. In this trial she had smooth ET for two (4A, 4B) embryos.

The third case was 37-year-old with previous failed 2 trials of ICSI with extreme difficulty in embryo transfer. in this trial there was failure of fertilization.

Informed written consent was taken from the patients after explaining the technique and its complications and that it is under trial.

After complete pituitary down regulation with the end of the withdrawal bleeding office hysteroscopy was done using Trophy scope ${ }^{\circledR}$ (Storz) with distension media (normal saline $0.9 \%$ ) to insert guide wire 4 Fr (Figure 1). Serial insertion of part of Nelton urinary catheter 12 and $14 \mathrm{Fr}$ across the inserted guide wire (Figure 2). Fix the $14 \mathrm{Fr}$ catheter with 2/0 prolene suture (Ethicon) to the cervix (Figure 3). This part of the catheter equal the

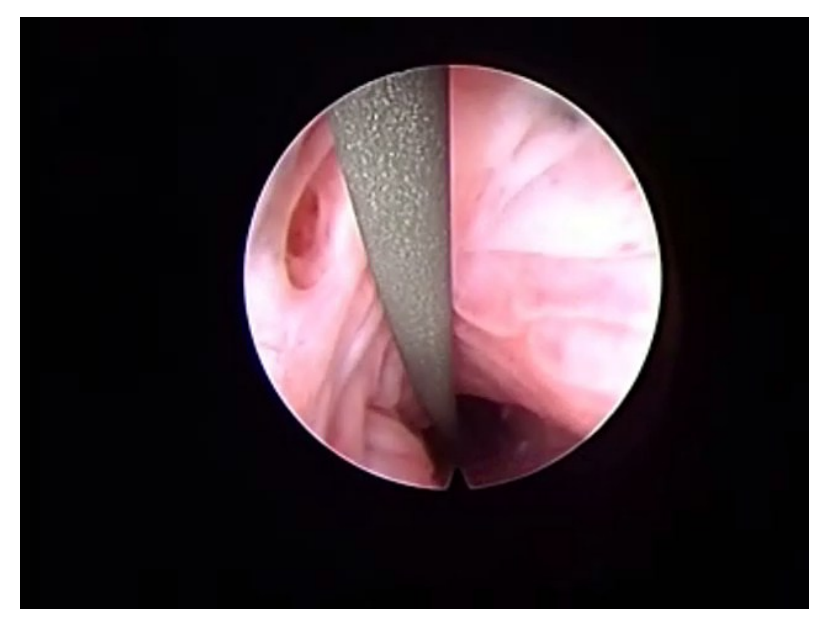

Figure 1. Insertion of the guide wire. 


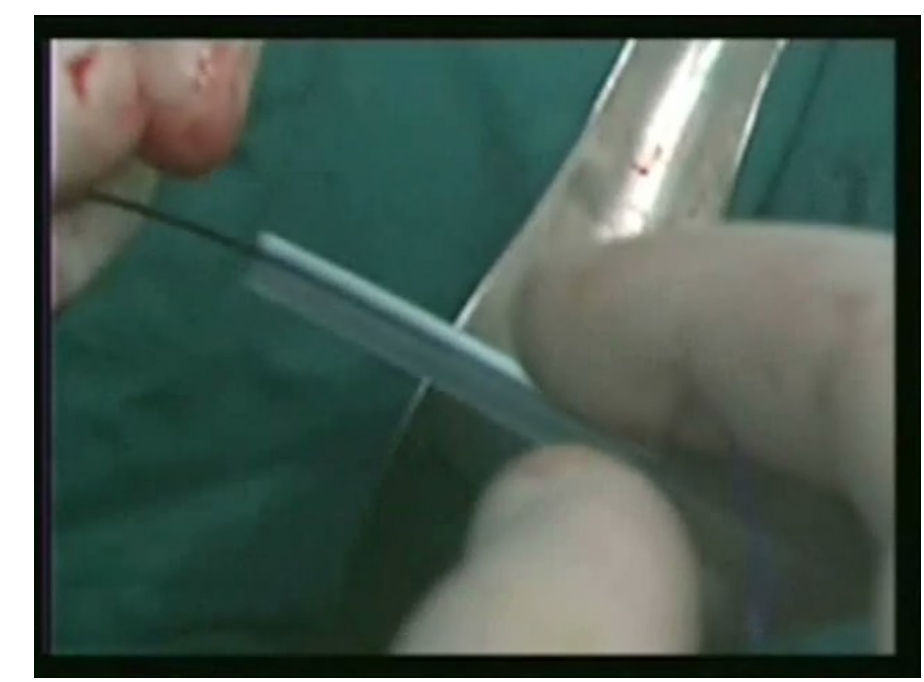

Figure 2. Serial insertion of the stents.

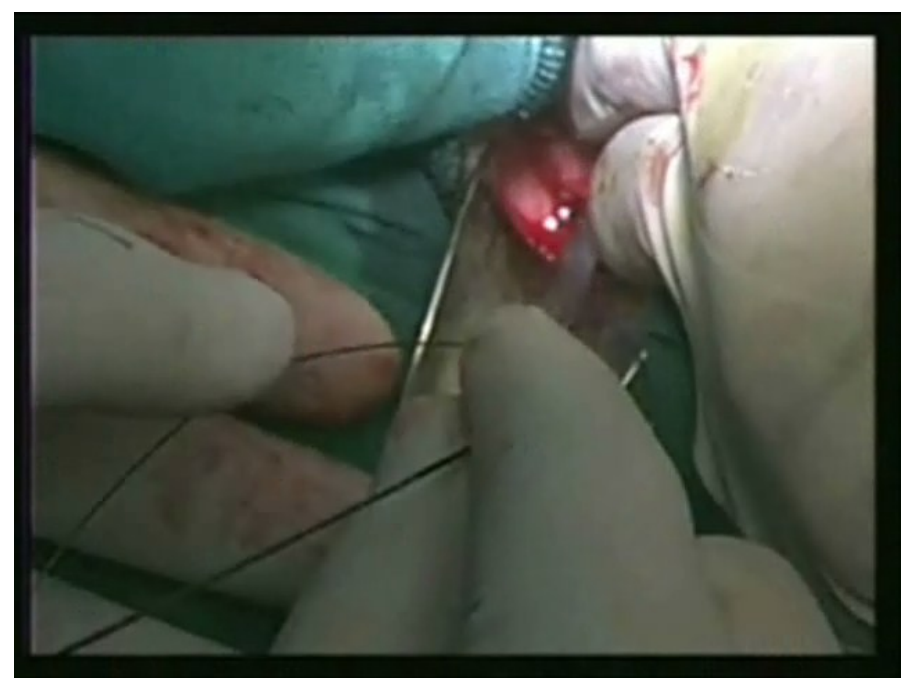

Figure 3. Insertion of the 14 Fr. catheter in the cervical canal.

distance between the external os and just above the internal os. Doxycycline $100 \mathrm{mg}$ was given twice daily for 7 days. On the day of embryo transfer repeated wash of the catheter using Ham's F10 media ${ }^{\circledR}$ (Irvine scientific) (Nutrient Mixture) composed of L-glutamine and sodium bicarbonate, Sterile-filtered, Endotoxin tested and Cell culture tested previously incubated to remove any discharge or any debris. Ultrasound guided embryo transfer by labotect ${ }^{\circledR}$ embryo transfer catheter and then the stent was removed.

\section{Discussion}

Difficult embryo transfer is a problem that affects the outcome of IVF and ICSI cases. In this technique, introducing the embryo transfer catheter through the stent was very easy without need for anesthesia, less invasive without any trauma to the endometrium near the time of embryo transfer. If compared to the other approaches used for difficult transfer it is easier.

Different maneuvers were tried before by changing the type of embryo transfer catheter type but this isn't helpful in all cases. Hysteroscopic shaving of the cervical canal was tried before to facilitate the transfer in cases with abnormal cervical canal [12]. This technique itself may cause more harm to the cervical canal as it may initiate more fibrosis and may lead to cervical trauma or incompetence. Cervical dilatation at the time of ovum pickup was adopted in some cases but with lower pregnancy rate as this may be associated with more uterine 
trauma near the time of transfer [10]. Zygote intrafallopian transfer was discussed as a tool for these cases but it is more invasive technique [8]. Also trans-myometrial embryo transfer is more invasive technique with myometrial trauma at the time of embryo transfer with lower pregnancy rate than transcervical approach [14].

The two problems which may be encountered are infection and the material of the catheter used. Patients with recurrent vaginitis or cervicitis may not be a suitable candidate for this technique. Also, vaginal and cervical culture and sensitivity must be done before insertion to avoid ascending uterine or pelvic infection. So, hysteroscopic guided insertion of cervical stent may be a tool in cases of difficult or failed embryo transfer due to abnormal cervical track. The small number of cases was the limitation to this technique assessment. So, further trial on wide scale may improve the outcome.

\section{Acknowledgements}

To the IVF laboratory staff members for their technical support.

\section{Disclosure Statement}

There is nothing to disclose.

\section{References}

[1] Buckett, W.M. (2003) A Meta-Analysis of Ultrasound-Guided versus Clinical Touch Embryo Transfer. Fertility and Sterility, 80, 1037-1041. http://dx.doi.org/10.1016/S0015-0282(03)01015-X

[2] Sallam, H.N. and Sadek, S.S. (2003) Ultrasound-Guided Embryo Transfer: A Meta-Analysis of Randomized Controlled Trials. Fertility and Sterility, 80, 1042-1046. http://dx.doi.org/10.1016/S0015-0282(03)01009-4

[3] Abou-Setta, A.M., Mansour, R.T., Al-Inany, H.G., Aboulghar, M.M., Aboulghar, M.A. and Serour, G.I. (2007) Among Women Undergoing Embryo Transfer, Is the Probability of Pregnancy and Live Birth Improved with UltrasoundGuidance than with Clinical Touch Alone? A Systemic Review and Meta-Analysis of Prospective Randomized Trials. Fertility and Sterility, 88, 333-341. http://dx.doi.org/10.1016/j.fertnstert.2006.11.161

[4] Brown, J.A., Buckingham, K., Abou-Setta, A. and Buckett, W. (2007) Ultrasound versus ‘Clinical Touch’ for Catheter Guidance during Embryo Transfer in Women. Cochrane Database of Systematic Reviews, 24, CD006107. http://dx.doi.org/10.1002/14651858.cd006107.pub2

[5] Sadek, S., Sallam, H., Agameya, A.-F., Sadek, S.S. and Sallam, H.N. (2004) Does a Difficult Embryo Transfer Affect the Results of IVF and ICSI?-A Meta-Analysis of Controlled. Fertility and Sterility, 81, 22. http://dx.doi.org/10.1016/j.fertnstert.2004.02.046

[6] Lesny, P., Killick, S.R. and Robinson, J. (1999) Transcervical Embryo Transfer as a Risk Factor for Ectopic Pregnancy. Fertility and Sterility, 72, 305-309. http://dx.doi.org/10.1016/S0015-0282(99)00226-5

[7] Marikinti, K., Mathews, T., Ball, J. and Brinsden, P. (2003) Ultrasound and Hysteroscopic Findings in Women with a History of Difficult Embryo Transfers. Fertility and Sterility, 80, 125. http://dx.doi.org/10.1016/S0015-0282(03)01201-9

[8] Rolla, E.D. (2010) Other Alternatives for Difficult Embryo Transfer. Fertility and Sterility, 94, e86. http://dx.doi.org/10.1016/j.fertnstert.2010.09.056

[9] Glatstein, I.Z., Pang, S.C. and McShane, P.M. (1997) Successful Pregnancies with the Use of Laminaria Tents before Embryo Transfer for Refractory Cervical Stenosis. Reproductive Science Center of Boston, 67, 1172-1174. http://dx.doi.org/10.1016/s0015-0282(97)81460-4

[10] McManus, J., McClure, N. and Traub, A.I. (2000) The Effect of Cervical Dilatation in Patients with Previous Difficult Embryo Transfer. Fertility and Sterility, 74, S159-S160. http://dx.doi.org/10.1016/s0015-0282(00)01179-1

[11] Yanushpolsky, E.H., Ginsburg, E.S., Fox, J.H. and Stewart, E.A. (2000) Transcervical Placement of a Malecot Catheter* after Hysteroscopic Evaluation Provides for Easier Entry into the Endometrial Cavity for Women with Histories of Difficult Intrauterine Inseminations and/or Embryo Transfers: A Prospective Case Series. Fertility and Sterility, 73, 402-405. http://dx.doi.org/10.1016/S0015-0282(99)00492-6

[12] Noyes, N. (1999) Hysteroscopic Cervical Canal Shaving: A New Therapy for Cervical Stenosis before Embryo Transfer in Patients Undergoing in Vitro Fertilization. Fertility and Sterility, 71, 965-966. http://dx.doi.org/10.1016/S0015-0282(99)00097-7

[13] Kilani, Z., Shaban, M. and Hassan, L.H. (2009) Live Birth after Hysteroscopic-Guided Embryo Transfer: A Case Report. Fertility and Sterility, 91, 2733.e1-2733. http://dx.doi.org/10.1016/j.fertnstert.2008.12.136

[14] Groutz, A., Lessing, J., Wolf, Y., Azem, F., Yovel, I. and Amit, A. (1997) Comparison of Transmyometrial and Transcervical Embryo Transfer in Patients with Previously Failed in Vitro Fertilization-Embryo Transfer Cycles and/or Cervical Stenosis. Fertility and Sterility, 67, 1073-1076. http://dx.doi.org/10.1016/S0015-0282(97)81441-0 